\title{
Study on the Surgical Treatment of Large Pituitary Adenomas
}

\author{
Xiaofei Fan ${ }^{1, a}$, Shuying Yue ${ }^{1, b}$ \\ ${ }^{1}$ Shandong Medical College, Jinan, Shandong, 250002 \\ ${ }^{a}$ email, ${ }^{b}$ email
}

Keywords: Surgical Treatment, Large Pituitary Adenomas, Medical Development Trend

\begin{abstract}
Pituitary adenoma is a common intracranial tumor, second only to glioblastoma and meningioma, accounting for about $10 \%$ of intracranial tumors. In recent years there has been an increasing trend. Hardy is divided into microadenomas $(<10 \mathrm{~mm})$, large adenomas $(11 \sim 40 \mathrm{~mm})$, giant adenomas ( $>40 \mathrm{~mm}$ ) according to imaging findings. According to the endocrine function can be divided into functional adenoma and nonfunctional adenoma. Functional adenoma is mainly prolactinoma, growth hormone adenoma, adrenocorticotropic hormone adenoma and so on. Pituitary adenomas mainly from the following aspects of harm to the human body: (1) excessive secretion or deficiency of pituitary hormones, causing metabolic disorders and organ damage; (2) tumor compression surrounding structures such as optic chiasm, optic nerve, cavernous sinus, hypothalamus A serious obstacle to the corresponding function. The early age of pituitary adenomas is young adults, which has important damage to the growth, development, labor ability and fertility of the patients, and cause a series of social psychological effects. Since the late 19th century, people's understanding of pituitary adenomas has been deepening, especially since the 1970s, with the development of modern endocrinology, pathology, imaging and microsurgery, the clinical and basic research of pituitary adenomas A lot of new progress, which deepened the understanding of the disease, improve the level of diagnosis and treatment.
\end{abstract}

\section{Introduction}

Selection criteria: 2002.11-2004.1 period of our hospital neurosurgical treatment of large and giant pituitary adenoma a total of 34 cases, using microsurgical techniques and endoscopic surgery with different surgical approach for treatment.

General information: 12 males and 22 females, aged 23-68 years, mean 35.5 years old. In the course of 10 days -18 years, an average of 3.6 years. All cases were confirmed by surgery and pathology. It includes 26 cases of large adenoma, 8 cases of giant adenoma.

Clinical manifestations: headache and other intracranial pressure increased in 13 cases; unilateral or bilateral visual loss in 23 cases; monocular or binocular vision defect in 22 cases; primary retinal head atrophy in 19 cases; menstrual disorders or amenorrhea in 13 cases; 5 cases of hypertrophy, concentric obesity in 2 cases; loss of libido in 1 case; polyuria in 1 case; goiter in 1 case. Among them, headache and other intracranial pressure increased as the first manifestation of 3 cases, visual dysfunction as the first manifestation of 21 cases, endocrine disorders as the first manifestation of 10 cases.

Tumor classification: the type of tumor based on clinical endocrine symptoms, blood hormone levels, pathology and immunohistochemistry to determine. 34 cases of functional pituitary adenoma (NFA) in 8 cases; functional adenoma (FA) in 26 cases. Including 3 cases of prolactin (PRL) tumor, 1 case of growth hormone (GH) adenoma, 4 cases of adrenocorticotropic hormone (ACTH) adenoma, 1 case of thyroid hormone (TSH) adenoma, 17 cases of mixed pituitary adenoma The

\section{Surgical Method}

In 34 cases of large and giant pituitary adenomas, the general is divided into two categories of transcranial and transsphenoidal surgery. (1) the tumor to the saddle was significantly expanded, and was dumbbell-shaped or lobulated, suggesting that the saddle is smaller, saddle on the tumor is not easy to saddle within the collapse; (2) tumor To the saddle, saddle or anterior cranial fossa 
expansion; (3) sphenoid sinus dysplasia such as methyl sphenoid sinus. Transsphenoidal approach indications include: (1) pituitary large adenoma, but the tumor is located in the saddle, and to the sphenoid sinus violations; (2) pituitary large adenoma, but the main tumor in the saddle, and saddle (3) patients with frail elderly, or suffering from severe organic disease and can not tolerate craniotomy.

Traditional transcranial surgery: the amount of the next approach to take the coronal incision, flap forward. The general line of the right amount of craniotomy, forehead bone flap should be as close as possible to the anterior cranial fossa, frontal if open to tightly closed. Along the orbital margin of the semi-curved cut dura mater, turned to the skull base with suture fixation in the periosteum. With the brain pressure plate gently lift the frontal lobe to reveal the lateral fissure, slowly release cerebrospinal fluid to reduce intracranial pressure, from outside and inside, by the shallow and deep gradually raised frontal lobe, exposed saddle area. Along the sphenoid crest gradually to the inside of the lateral lateral root, in the former bed to see the lateral optic nerve, optic nerve outside the ipsilateral carotid artery. The inside of the optic nerve is the saddle of the tumor. At the same time visible contralateral optic nerve. The structure of the surface covered with a layer of arachnoid, with a micro-scissors or micro-stripping sub-arachnoid, fully revealed between the two sides of the optic nerve saddle diaphragm, frontal lobe with wet cotton to protect the placement of automatic pressure plate. Between the two sides of the optic nerve, with bipolar coagulation saddle diaphragm dural, routine puncture to exclude aneurysms, with a knife or scissors in the saddle dural on the window, the window should not exceed the scope of both sides of the optic nerve and its Behind the crossover front. When the tumor is soft, the tumor can self-emit, and the suction can be sucked. With the suction device to suck some of the saddle after the tumor, and then the appropriate size of the curettage into the saddle within the curettage saddle tumor, while gently scraping with suction suction. Saddle tumor removal after thorough, visible dark yellow posterior pituitary tissue. With gelatin sponge pressure saddle around the bleeding. General tumor resection completely after the saddle to stop bleeding easier. On the saddle on the part of the tumor, the first cyst tumor resection, and then gradually remove the tumor. Lift the tumor, along the brain tissue between the arachnoid interface separation, pay attention to the protection of cerebrovascular structure. Sometimes into the saddle on the huge tumor and hypothalamus, pituitary stalk and anterior cerebral artery adhesion is very tight, this time can not be forced to remove the tumor to avoid damage to the structure. Another way to pass the way is through the vertical approach. The approach also takes the coronal incision and turns the flap forward. And the lower approach is different from the bone flap near the skull base on both sides of about $4 \times 4 \mathrm{~cm}$ small bone window. After removal of the bone flap, suture the sagittal sinus and cut it with the brain sickle. To the brain pressure plate separated from the inside of the brain, along the longitudinal separation of the saddle area has been reached. Tumor resection is the same as the frontal approach. The approach for the frontal traction is small, the partial removal of the saddle easier.

Endoscopic nasal - sphenoid sinus surgery. Patients take supine position, head drooping $30^{\circ}$, to the side of the surgeon $30^{\circ}$ rotation. Facial skin with $05 \%$ iodophor disinfection. Nasal mucosa with 0 05\% povidone-iodine gauze packing disinfection. Apply a sterile towel, only exposed to the nasal spine and bilateral nostrils. Remove the iodophor gauze, under the guidance of endoscopy, the adrenaline saline soaked sliver along the total nasal passages to the butterfly screen crypt, contraction of the turbinate, total nasal passages and butterfly screen crypts, nasal stenosis Can be dislocation or partial resection in the rear of the turbinate, fully exposed butterfly screen crypt, open surgery space, middle turbinate stump bleeding, available bipolar coagulation coagulation hemostasis, adrenaline cotton sliver to stop bleeding. In the butterfly sieve to find the sphenoid sinus opening, along the sphenoid sinus opening open sphenoid sinus, the lower wall, to the diameter of $2 \mathrm{~cm}$. Into the sphenoid sinus, the removal of the bottom of the bone to the bone window diameter $10 \mathrm{~cm} \sim 15 \mathrm{~cm}$. After burning the saddle at the end of the dura mater, with a special slim knife "ten" word cut, endoscopic lighting, intermittent into the saline surgery field, with the suction device, take the tumor clamp, scraping ring resection of the tumor, most of the resection After the tumor, $30^{\circ}$ endoscopy into the tumor cavity, observe the residual tumor and bleeding point, under 
direct vision to further remove the saddle, saddle residual tumor, while identifying the bleeding point, coagulation or gelatin sponge pressure to stop bleeding, tumor resection satisfaction , The cavity filled with gelatin sponge, coated with gelatin sponge coated with biological glue repair saddle bottom, or with the beginning of surgery to remove the middle turbinate tissue, trimmed with biological glue, sticky in the saddle at the end of the defect, repair saddle at the end. After surgery, only in the side of the nasal cavity to fill a Vaseline gauze.

\section{Treatment Results}

In 34 cases of large and giant pituitary adenoma, the subordinate approach in 3 cases; through the longitudinal approach in 14 cases; the pter point approach in 8 cases; oral nose butterfly approach in 7 cases; supraorbital keyhole One case of endoscopic transnasal transsphenoidal surgery in 1 case. According to intraoperative microscopic findings and postoperative CT or MRI findings, total tumor in 31 cases, nearly 3 cases of total resection. Preoperative visual field damage in 23 cases, 20 cases of 1-3 days after surgery began to improve, no change or increased in 3 cases. 5 cases of preoperative lactation, 4 cases of lactation after surgery, 1 case was lower than before surgery. A sudden has loss of urine in 9 cases, cerebrospinal fluid rhinorrhea in 2 cases, 2 cases of water and electrolyte disorders, are in a few days after the self-healing. Pituitary function in 3 cases is postoperative taking thyroxine tablets and strong pine to improve the posterior pituitary function. Saddle hematoma has 1 case, 5 days after surgery craniotomy hematoma evacuation. The group of 19 patients were followed up, the time of March -1, an average of 6 months. It includes visual field of vision, blood hormone levels, imaging and so on. 14 patients received radiotherapy. Visual acuity improved compared with preoperative 16 cases. No change or exacerbation in 3 cases. Acromegaly hypertrophy in 5 cases, postoperative symptoms were reduced to varying degrees. 2 cases of cardiac obesity were varying degrees of weight loss after surgery. 26 cases of functional pituitary adenomas were followed up in 17 cases, blood hormone levels returned to normal in 13 cases, compared with preoperative reduction in 4 cases. 2 cases of tumor recurrence, were gamma-knife treatment.

The surgical treatment of large and giant pituitary adenomas. Methods Thirty-four cases of large and giant pituitary adenomas were treated with subfrontal approach (3 cases); transversal approach (14 cases); pterional approach (8 cases) 7 cases); supraorbital keyhole approach (1 case), endoscopic transnasal transsphenoidal surgery (1 case) implementation of treatment. Results According to the findings of intraoperative microscope and CT or MRI findings, 31 cases of pituitary adenoma resection, 3 cases of total resection, no obvious postoperative complications, no deaths. This shows that microsurgery and endoscopic surgery is a good way to treat pituitary adenomas.

\section{References}

[1] Zhou Tengyuan, Chen Laizhao. Pituitary adenoma diagnosis and treatment of the status quo and progress [J]. Chinese Journal of Clinical Medicine, 2016 (08)

[2] Peng Song. Talking about the treatment of pituitary adenoma [J]. Chinese Journal of Continuing Education, 2015 (14)

[3] Li Gan, Li Qiang, Ding Yongzhong, Ren Jun, Zhang Hailin. Study on the relationship between stromal cell-derived factor-1 and pituitary adenoma invasion [J]. Health Vocational Education, $2013(21)$

[4] Ding Zhemin, Lu Xiaojie, Wang Qing, Li Bing, Miao Yifeng. Expression and Significance of Folate Receptor $\alpha$ in Pituitary Adenomas [J]. Chinese Journal of Minimally Invasive Neurosurgery, 2011 (2)

[5] Fei Yi. Pituitary adenoma and abnormal signal transduction mechanism [J]. Chinese Journal of Tropical Medicine, 2012 (03) 